


\title{
Heegner Points and Elliptic Curves of Large Rank over Function Fields
}

\author{
HENRI DARMON
}

\begin{abstract}
This note presents a connection between Ulmer's construction [Ulm02] of non-isotrivial elliptic curves over $\mathbb{F}_{p}(t)$ with arbitrarily large rank, and the theory of Heegner points (attached to parametrisations by Drinfeld modular curves, as sketched in Section 3 of Ulmer's article (see page ??). This ties in the topics in Section 4 of that article more closely to the main theme of this volume.
\end{abstract}

A review of the number field setting. Let $K$ be a quadratic imaginary extension of $F=\mathbb{Q}$, and let $E_{/ \mathbb{Q}}$ be an elliptic curve of conductor $N$. When all the prime divisors of $N$ are split in $K / F$, the Heegner point construction (in the most classical form that is considered in [GZ], relying on the modular parametrisation $X_{0}(N) \longrightarrow E$ ) produces not only a canonical point on $E(K)$, but also a norm-coherent system of such points over all abelian extensions of $K$ which are of "dihedral type". (An abelian extension $H$ of $K$ is said to be of dihedral type if it is Galois over $\mathbb{Q}$ and the generator of $\operatorname{Gal}(K / \mathbb{Q})$ acts by -1 on the abelian normal subgroup $\operatorname{Gal}(H / K)$.) The existence of this construction is consistent with the Birch and Swinnerton-Dyer conjecture, in the following sense: an analysis of the sign in the functional equation for $L(E / K, \chi, s)=L(E / K, \bar{\chi}, s)$ shows that this sign is always equal to -1 , for all complex characters $\chi$ of $G:=$ $\operatorname{Gal}(H / K)$. Hence

$$
L(E / K, \chi, 1)=0 \quad \text { for all } \chi: G \longrightarrow \mathbb{C}^{\times} .
$$

The product factorisation

$$
L(E / H, s)=\prod_{\chi} L(E / K, \chi, s)
$$

implies that

$$
\operatorname{ord}_{s=1} L(E / H, s) \geq[H: K]
$$


so that the Birch and Swinnerton-Dyer conjecture predicts that

$$
\operatorname{rank}(E(H)) \stackrel{?}{\geq}[H: K] \text {. }
$$

In fact, the $G$-equivariant refinement of the Birch and Swinnerton-Dyer conjecture leads one to expect that the rational vector space $E(H) \otimes \mathbb{Q}$ contains a copy of the regular representation of $G$.

It is expected in this situation that Heegner points account for the bulk of the growth of $E(H)$, as $H$ varies over the abelian extensions of $K$ of dihedral type. For example we have:

LEMMA 1. If $\operatorname{ord}_{s=1} L(E / H, s) \leq[H: K]$, then the vector space $E(H) \otimes \mathbb{Q}$ has dimension $[H: K]$ and is generated by Heegner points.

Proof. For $V$ any complex representation of $G$, let

$$
V^{\chi}:=\{v \in V \quad \text { such that } \sigma v=\chi(\sigma) v \text {, for all } \sigma \in G\} .
$$

Since equality is attained in (1), it follows that each $L(E / K, \chi, s)$ vanishes to order exactly one at $s=1$. Zhang's extension of the Gross-Zagier formula to $L$ functions $L(E / K, s)$ twisted by (possibly ramified) characters of $G$ [Zh01] shows that

$$
\operatorname{dim}_{\mathbb{C}}\left(H P^{\chi}\right)=1,
$$

where $H P$ denotes the subspace of $E(H) \otimes \mathbb{C}$ generated by Heegner points. Theorem 2.2 of [BD90], whose proof is based on Kolyvagin's method, then shows that

$$
\operatorname{dim}_{\mathbb{C}}\left((E(H) \otimes \mathbb{C})^{\chi}\right) \leq 1
$$

The result follows directly from (3) and (4).

0.1. The case $F=\mathbb{F}_{q}(u)$. As explained in Section 3 of [Ulm03], the Heegner point construction can be adapted to the case where $\mathbb{Q}$ is replaced by the rational function field $\mathbb{F}_{q}(u)$.

The basic idea of our construction is to start with an elliptic curve $E_{0}$ defined over $\mathbb{F}_{p}(u)$, and produce a Galois extension $H$ of $\mathbb{F}_{q}(u)$ (for some power $q$ of $p$ ) such that

(i) the Galois group of $H$ over $\mathbb{F}_{q}(u)$ is isomorphic to a dihedral group of order $2 d$;

(ii) $H$ satisfies a suitable Heegner hypothesis relative to $E_{0}$ over $\mathbb{F}_{q}(u)$ so that the Birch and Swinnerton-Dyer conjecture implies an inequality like (2);

(iii) $H$ is the function field of a curve of genus 0 , say $H=F_{q}(t)$, so that $E_{0}$ yields a curve $E$ over $\mathbb{F}_{p}(t)$ which acquires rank at least $d$ over $\mathbb{F}_{q}(t)$.

A further argument is then made to show that the rank of $E$ remains large over $\mathbb{F}_{p}(t)$, provided suitable choices of $d$ and $q$ have been made.

To illustrate the method, let $p$ be an odd prime and let $F_{0}$ be the field $\mathbb{F}_{p}(u)$, with $u$ an indeterminate. Let $K_{0}=\mathbb{F}_{p}(v)$ be the quadratic extension of $F_{0}$ 
defined by $v+v^{-1}=u$. Choose an element $u_{\infty} \in \mathbb{P}_{1}\left(\mathbb{F}_{p}\right)$ such that the place $\left(u-u_{\infty}\right)$ is inert in $K_{0}$. (Such a $u_{\infty}$ always exists when $p>2$.) The chosen place $u_{\infty}$ will play the role in our setting of the archimedean place of $\mathbb{Q}$ in the previous discussion. Note that $K_{0} / F_{0}$ becomes a quadratic "imaginary" extension with this choice of place at infinity, and that this continues to hold when $\mathbb{F}_{p}$ is replaced by $\mathbb{F}_{q}$ with $q=p^{m}$, provided that $m$ is odd.

Let $E=E_{u}$ be an elliptic curve over $F_{0}$ having split multiplicative reduction at $u_{\infty}$. Let $\mathcal{E}$ denote the Néron model of $E$ over the subring $\mathcal{O}=\mathbb{F}_{p}\left[\frac{1}{u-u_{\infty}}\right]$ and let $N$ denote its arithmetic conductor, viewed as a divisor of $\mathbb{P}_{1}-\left\{u_{\infty}\right\}$. Suppose that

$$
\text { all prime divisors of } N \text { are split in } K_{0} / F_{0} \text {, }
$$

which is the analogue of the classical Heegner hypothesis in our function field setting.

Finally, given any integer $d$, let $o_{d}$ be the order of $p$ in $(\mathbb{Z} / d \mathbb{Z})^{\times}$. Assume that

$$
\text { the integer } o_{d} \text { is odd. }
$$

We then set $q=p^{o_{d}}$ and consider the extensions

$$
F=\mathbb{F}_{q}(u) ; \quad K=\mathbb{F}_{q}(v) ; \quad H=\mathbb{F}_{q}(t), \text { with } v=t^{d} .
$$

Note that $H / K$ is an abelian extension with Galois group $G=\mathrm{Gal}(H / K)$ isomorphic to $\mu_{d}\left(\mathbb{F}_{q}\right) \simeq \mathbb{Z} / d \mathbb{Z}$, and that this extension is of dihedral type, relative to the ground field $F$. Therefore the analysis of signs in functional equations that was carried out to conclude (1) carries over, mutatis mutandis, to prove the following.

Proposition 2. Assume the Birch and Swinnerton-Dyer conjecture over function fields. Then the rank of $E(H)$ is at least d. More precisely,

$$
\operatorname{dim}_{\mathbb{C}}\left((E(H) \otimes \mathbb{C})^{\chi}\right) \geq 1, \quad \text { for all } \chi: G \longrightarrow \mathbb{C}^{\times} .
$$

One also wants to estimate the rank of $E$ over the field $H_{0}:=\mathbb{F}_{p}(t)$. Let $\tilde{G}=\operatorname{Gal}\left(H / K_{0}\right)$; then $\tilde{G}$ is the semi-direct product $G \times\langle f\rangle$, where $\langle f\rangle \subset$ $(\mathbb{Z} / d \mathbb{Z})^{\times}$is the cyclic group of order $o_{d}$ generated by the Frobenius element $f \in \operatorname{Gal}\left(H / H_{0}\right)=\operatorname{Gal}\left(\mathbb{F}_{q} / \mathbb{F}_{p}\right)$, which acts by conjugation on the abelian normal subgroup $G=\mu_{d}\left(\mathbb{F}_{q}\right)$ in the natural way. Since $E$ is defined over $K_{0}$ (and even over $F_{0}$ ), the space $V:=E(H) \otimes \mathbb{C}$ is a complex representation of $\tilde{G}$, and one may exploit basic facts about the irreducible representations of such a semidirect product to obtain lower bounds for $E(H)^{f=1}=E\left(\mathbb{F}_{p}(t)\right)$. More precisely, suppose that the character $\chi$ of $G$ is one of the $\phi(d)$ faithful characters of $G$. Proposition 2 asserts that the space $V^{\chi}$ contains a non-zero vector $v_{\chi}$. Note that $V^{\chi}$ is not preserved by the action of $f$, which sends $V^{\chi}$ to $V^{\chi^{p}}$. Because of this, the vectors $v_{\chi}, f v_{\chi}, \ldots, f^{o_{d}-1} v_{\chi}$ are linearly independent since they belong to different eigenspaces for the action of $G$. Hence the vector

$$
v_{[\chi]}=v_{\chi}+f v_{\chi}+\cdots+f^{o_{d}-1} v_{\chi}
$$


is non-zero and belongs to $V^{f=1}=E\left(H_{0}\right) \otimes \mathbb{C}$. Futhermore the $v_{[\chi]}$ are linearly independent, as $\chi$ ranges over the $f$-orbits of faithful characters of $G$. Hence

$$
\operatorname{rank}\left(E\left(\mathbb{F}_{p}(t)\right) \geq \phi(d) / o_{d} .\right.
$$

By taking into account the contributions coming from all the characters (and not just the faithful ones) one can obtain the following stronger estimate.

Proposition 3. Assume the Birch and Swinnerton-Dyer conjecture over function fields. Then

$$
\operatorname{rank}\left(E\left(\mathbb{F}_{p}(t)\right) \geq \sum_{e \mid d} \frac{\phi(e)}{o_{e}} \geq \frac{d}{o_{d}} .\right.
$$

Proof. A complex character $\chi$ of $G$ is said to be of level $e$ if its image is contained in the group $\mu_{e}$ of $e$ th roots of unity in $\mathbb{C}$ and in no smaller subgroup. Clearly the level $e$ of $\chi$ is a divisor of $d$, the order $o_{e}$ of $p$ in $(\mathbb{Z} / e \mathbb{Z})^{\times}$divides $o_{d}$, and there are exactly $\phi(e)$ distinct characters of $G$ of level $e$. Note also that if $\chi$ is of level $e$, then $f^{o_{e}}$ maps $V^{\chi}$ to itself. The same reasoning used to prove Proposition 2, but with $d$ replaced by $e$, and $q$ by $p^{o_{e}}$, shows that (under the Birch and Swinnerton-Dyer assumption)

$$
V^{\chi} \quad \text { contains a non-zero vector fixed by } f^{o_{e}} \text {. }
$$

If $v_{\chi}$ is such a vector, then just as before the vectors

$$
v_{[\chi]}=v_{\chi}+f v_{\chi}+\cdots+f^{o_{e}-1} v_{\chi}
$$

form a linearly independent collection of $\phi(e) / o_{e}$ vectors in $E\left(\mathbb{F}_{p}(t)\right) \otimes \mathbb{C}$, as $\chi$ ranges over the $f$-orbits of characters of $G$ of level $e$. Summing over all $e$ dividing $d$ proves the first inequality in (7). The second is obtained by noting that

$$
\sum_{e \mid d} \frac{\phi(e)}{o_{e}} \geq \frac{1}{o_{d}} \sum_{e \mid d} \phi(e)=\frac{d}{o_{d}} .
$$

REMARKs. 1. It is instructive to compare the bound (7) with the formula for the rank of Ulmer's elliptic curves which is given in Theorem 4.2.1 of [Ulm03]. 2. Note that the expression which appears on the right of (7) can be made arbitrarily large by setting $d=p^{n}-1$ with $n$ odd, so that $o_{d}=n$.

Some examples. Elliptic curves satisfying the Heegner assumptions of the previous section are not hard to exhibit explicitly. For example, suppose for notational convenience that $p$ is congruent to 3 modulo 4 , and let $E[u]$ be a non-isotrivial elliptic curve over $\mathbb{F}_{p}(u)$ having good reduction everywhere except at $u=0,1$ and $\infty$, and having split multiplicative reduction at $u_{\infty}=0$. There are a number of such curves, for example:

(i) An (appropriate twist of a) "universal" elliptic curve over the $j$-line in characteristic $p \neq 2,3$, with $u=1728 / j$;

(ii) A "universal" curve over $X_{0}(2)$, or over $X_{0}(3)$; 
(iii) The Legendre family $y^{2}=x(x-1)(x-u)$ (corresponding to a universal family over the modular curve $X(2))$.

(iv) The curve $y^{2}+x y=x^{3}-u$ that is used in [Ulm03], in which the parameter space has no interpretation as a modular curve.

Choosing any parameter $\lambda$ in $\mathbb{F}_{p}-\{0, \pm 1\}$, we see that the curve $E\left[\frac{u}{\lambda+\lambda^{-1}}\right]$ over $\mathbb{F}_{p}(u)$ satisfies all the desired properties, since two of the places $u=\infty$ and $\lambda+\lambda^{-1}$ dividing the conductor of $E$ are split in $K / F$, while the third place $u=0$, which lies below $v= \pm i$, is inert in $K / F$. (This is where the assumption $p \equiv 3$ (mod 4$)$ is used.) Hence Proposition 3 implies

Corollary 4. Assume the Birch and Swinnerton-Dyer conjecture for function fields. Let $E[u]$ be any of the curves over $\mathbb{F}_{p}(u)$ listed above, and let $\lambda$ be any element in $\mathbb{F}_{p}-\{0, \pm 1\}$. Then the curve

$$
E\left[\frac{t^{d}+t^{-d}}{\lambda+\lambda^{-1}}\right]
$$

has rank at least $d / o_{d}$ over $\mathbb{F}_{p}(t)$.

Dispensing with the Birch and Swinnerton-Dyer hypothesis. It may be possible, at least for some specific choices of $E[u]$ and of $d$, to remove the Birch and Swinnerton-Dyer assumption that appears in corollary 4, since the notion of Heegner points which motivated Proposition 2 also suggests a possible construction of a (hopefully, sufficiently large) collection of global points in $E(H)$. To produce explicit examples where the module $H P$ generated by Heegner points in $E(H)$ has large rank, it may not be necessary to invoke the full strength of the theory described in Section 3 of [Ulm03] since quite often the mere knowledge that the Heegner point on $E(K)$ is of infinite order is sufficient to gain strong control over the Heegner points that appear in related towers. It appears worthwhile to produce explicit examples where Propositions 2 and 3 can be made unconditional thanks to the Heegner point construction.

Remark. Crucial to the construction in this note is the fact that $\mathbb{P}_{1}$ has a large automorphism group, containing dihedral groups of arbitrarily large order. Needless to say, this fact breaks down when $\mathbb{F}_{p}(u)$ is replaced by $\mathbb{Q}$, which has no automorphisms. In this setting Heegner points are known to be a purely "rank one phenomenon", and are unlikely to yield any insight into the question of whether the rank of elliptic curves over $\mathbb{Q}$ is unbounded or not.

Remarks on Ulmer's construction. Let $d$ be a divisor of $q+1$, where $q=p^{n}$. The curve

$$
E_{d}: y^{2}+x y=x^{3}-t^{d}
$$

studied in Theorem 4.2.1 of [Ulm03] is a pullback of the curve

$$
E_{0}: y^{2}+x y=x^{3}-u
$$


by the covering $\mathbb{P}_{1} \rightarrow \mathbb{P}_{1}$ given by $t \mapsto u:=t^{d}$, a covering that becomes Galois (abelian) over $\mathbb{F}_{q^{2}}$. It is not hard on the other hand to see that the curve $E_{d}$ does not arise as a pullback via any geometrically connected dihedral covering $\mathbb{P}_{1} \rightarrow \mathbb{P}_{1}$. However, one may set

$$
F=\mathbb{F}_{q}(u), \quad K=\mathbb{F}_{q^{2}}(u), \quad H=\mathbb{F}_{q^{2}}(t), \text { with } u=t^{d} .
$$

The congruence $q \equiv-1(\bmod d)$ implies that $\operatorname{Gal}(H / F)$ is a dihedral group of order $2 d$. Hence is becomes apparent a posteriori that the curves of [Ulm02] can be approached by a calculation of the signs in functional equations for the $L$-series of $E_{0}$ over $K$ twisted by characters of $\operatorname{Gal}(H / K)$. (See the remarks in sec. 4.3 of [Ulm03] for further details on this calculation and its close connection with the original strategy followed in [Ulm02].)

It should be noted that the elliptic curves produced in our Corollary 4 have smaller rank-to-conductor ratios than the curves $E_{d}$ in Theorem 4.2.1 of [Ulm03], which are essentially optimal in this respect.

\title{
References
}

[BD90] Massimo Bertolini and Henri Darmon, "Kolyvagin's descent and Mordell-Weil groups over ring class fields", J. Reine Angew. Math. 412 (1990), 63-74.

[GZ] Benedict Gross and Don Zagier, "Heegner points and derivatives of $L$-series", Invent. Math. 84:2 (1986), 225-320.

[Ulm02] Douglas Ulmer, "Elliptic curves with large rank over function fields", Ann. of Math. (2) 155:1 (2002), 295-315.

[Ulm03] Douglas Ulmer, "Elliptic curves and analogies between number fields and function fields", pp. 285-315 in Heegner Points and Rankin L-Series, edited by Henri Darmon and Shou-Wu Zhang, Math. Sci. Res. Inst. Publications 49, Cambridge U. Press, New York, 2004.

[Zh01] Shou-Wu Zhang, "Gross-Zagier formula for GL 2 ", Asian J. Math. 5:2 (2001), 183-290.

\author{
HENRI DARMON \\ Mathematics and Statistics Department \\ MCGILl UNIVERSITY \\ 805 Sherbrooke W. \\ Montreal, Quebec \\ Canada H3A $2 \mathrm{~K} 6$ \\ darmon@math.mcgill.ca
}

